Article

\title{
Correlations between Garnet Species and Vibration Spectroscopy: Isomorphous Substitution Implications
}

\author{
Weiwei $\mathrm{Li}^{1,2}{ }^{1}$, Jinyu Zheng ${ }^{1}\left(\mathbb{D}\right.$, Jingcheng Pei ${ }^{1}$, Xing $\mathrm{Xu}^{1}$ and Tao Chen ${ }^{1,3, *}$ \\ 1 Institute of Gemology, China University of Geosciences, Wuhan 430074, China; \\ mg20290006@smail.nju.edu.cn (W.L.); gemfisher@cug.edu.cn (J.Z.); peijc@cug.edu.cn (J.P.); \\ xmzgic@163.com (X.X.) \\ 2 State Key Laboratory of Mineral Deposit Research, School of Earth Sciences and Engineering, \\ Nanjing University, Nanjing 210023, China \\ 3 State Key Laboratory of Geological Processes and Mineral Resources, China University of Geosciences, \\ Wuhan 430074, China \\ * Correspondence: chentao@cug.edu.cn; Tel.: +86-189-7161-6137
}

Citation: Li, W.; Zheng, J.; Pei, J.; Xu, $\mathrm{X}$; Chen, T. Correlations between Garnet Species and Vibration Spectroscopy: Isomorphous Substitution Implications. Crystals 2022, 12, 104. https://doi.org/ $10.3390 /$ cryst12010104 Academic Editors: Taijin Lu, Fei Liu and Tingting $\mathrm{Gu}$

Received: 4 December 2021

Accepted: 2 January 2022

Published: 13 January 2022

Publisher's Note: MDPI stays neutral with regard to jurisdictional claims in published maps and institutional affiliations.

Copyright: (C) 2022 by the authors. Licensee MDPI, Basel, Switzerland. This article is an open access article distributed under the terms and conditions of the Creative Commons Attribution (CC BY) license (https:// creativecommons.org/licenses/by/ $4.0 /$ )

\begin{abstract}
Garnet has many species because of its common isomorphism. In this study, a suite of 25 natural gem-quality garnets, including pyrope, almandine, spessartine, grossular, and andradite, were examined by standard gemological testing, LA-ICP-MS, FTIR, and Raman analysis. Internal stretching and bending vibrations of the $\mathrm{SiO}_{4}$-tetrahedra of garnet exhibit correlate with the type of cations in garnet's dodecahedral position (A site) and octahedral position (B site). FTIR and Raman spectra showed that with the increase of the radius of $\mathrm{Mg}^{2+}, \mathrm{Fe}^{2+}, \mathrm{Mn}^{2+}$, and $\mathrm{Ca}^{2+}$ in $\mathrm{A}$ site, or the unit cell volumes of pyrope, almandine, spessartine, and grossular, the spectral peaks of $\mathrm{Si}-\mathrm{O}_{\mathrm{str}}$ and $\mathrm{Si}-\mathrm{O}_{\text {bend }}$ modes shift to low wavenumber. Because of the largest cations both in A site $\left(\mathrm{Ca}^{2+}\right)$ and in $\mathrm{B}$ site $\left(\mathrm{Fe}^{3+}\right)$, andradite exhibited the lowest wavenumber of $\mathrm{Si}-\mathrm{O}_{\text {str }}$ and $\mathrm{Si}-\mathrm{O}_{\text {bend }}$ modes of the five garnet species. Therefore, garnet has correlations between chemical composition and vibration spectroscopy, and Raman or IR spectroscopy can be used to precisely identify garnet species.
\end{abstract}

Keywords: isomorphous substitution; garnet; LA-ICP-MS; FTIR; Raman

\section{Introduction}

The popular garnet family is one of the most prolific mineral groups and branches into a spectrum of colors and gem types [1,2]. The garnets used in gems are the common silicate garnets with relatively simple chemical compositions [3]. They crystallize in the highly symmetric cubic structure (space group $I a 3 d$ ) and are represented by the general formula $\mathrm{A}_{3} \mathrm{~B}_{2}\left[\mathrm{SiO}_{4}\right]_{3}$. The garnets belong to two subgroups and six end-members (species), known as the pyralspite subgroup (pyrope-almandine-spessartine; $\mathrm{B}=\mathrm{Al}^{3+}$ and $\mathrm{A}=\mathrm{Mg}^{2+}$, $\mathrm{Fe}^{2+}$, and $\mathrm{Mn}^{2+}$, respectively) and the ugrandite subgroup (grossular-andradite-uvarovite; $\mathrm{A}=\mathrm{Ca}^{2+}$ and $\mathrm{B}=\mathrm{Al}^{3+}, \mathrm{Fe}^{3+}$ and $\mathrm{Cr}^{3+}$, respectively) $[3,4]$. The really end-member garnet is uncommon because of broad chemical substitution [5].

Garnets have been widely studied by many gemologists, such as the cause of color, localities, and the typical inclusions of different garnet types, for example, in [4,6-14]. Commonly, the garnet types are determined using standard gemological methods, such as color, refractive indices (RI), specific gravity (SG), and absorption lines over the visible energy range by hand spectroscope $[1,9,15]$. However, because of complex isomorphous substitution, the RI and SG values of some garnets deviate from the range of relative endmembers. Such garnets cannot be easily determined by standard gemological methods [1].

Garnet offers an excellent system to study the vibrational spectroscopic properties of silicate structures because of its high symmetry and chemical substitution. A series of compositional different end-members gives chance to investigate changes in the spectra with changing chemistry $[1,16]$. The correlation between the Raman or infrared (IR) spectra 
and compositional changes have been investigated usually by using synthetic binary garnet samples with regularly changing compositions, such as the pyrope-grossular series [17,18], grossular-uvarovite series [19], and skiagite-andradite and skiagite-almandine series [20]. Adamo et al. discussed the correlations among gemological properties, chemical composition, and IR vibrational frequencies in a suite of nature gem-quality garnets [1]. They proposed that the IR active bands over the $1150-800$ and $650-450 \mathrm{~cm}^{-1}$ ranges allow discrimination between pyralspite and ugrandite subgroups. However, identification of the end-members or species of garnets by vibrational spectra has not been proposed so far.

In the present paper, a suite of gem-quality garnets, including pyrope, almandine, spessartine, grossular, and andradite, were studied for the correlations among gemological properties, composition, and spectroscopic features. The distinction of garnet species by Raman and IR spectra was proposed. It provides a detailed set of spectroscopic data to help the precise identification of garnet species without composition analysis.

\section{Materials and Methods}

A total of 25 gem-quality faceted garnet samples, covering all the main subgroups of garnet, were selected to be studied for this study (Figure 1 and Table 1). UV fluorescence was detected by UV5000XL (Skyray, Suzhou, China). The RI was measured by refractometer (GIC, Wuhan, China, myopia method for faceted samples and hyperopia method for arc samples), and SG was determined hydrostatically (GIC, Wuhan, China). The above experiments were performed at the Gemological Institute, China University of Geosciences (Wuhan).

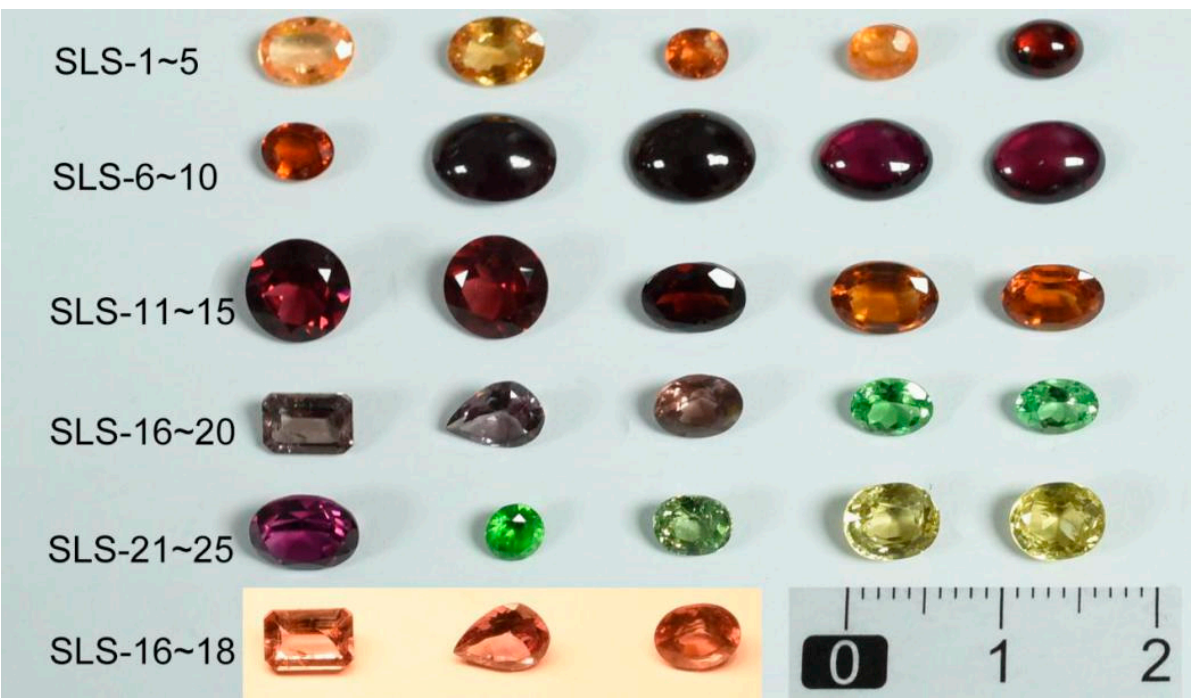

Figure 1. The studied gem garnet samples including spessartine (SLS-1, 3 to 6 and 16 to 18), grossular (SLS-2, 14, 15, 19, 20, 24 and 25), almandine (SLS-7 to 10), pyrope (SLS-11 to 13 and 21), andradite (SLS-22 and 23). Samples SLS-16 18 are Color-change garnets, were also observed in incandescent light and shown in the left bottom. The others were observed in daylight. Photos by T. Chen. 
Table 1. General gemological properties of the twenty-five garnet samples.

\begin{tabular}{|c|c|c|c|c|c|c|}
\hline $\begin{array}{c}\text { Sample } \\
\text { No. }\end{array}$ & $\begin{array}{l}\text { Weight } \\
\text { (ct) }\end{array}$ & SG & RI & Color & UVF * & Properties \\
\hline SLS-1 & 0.755 & 3.872 & $\mathrm{NR}^{*}$ & light-orange & $\mathrm{IF}^{*}$ & spessartine \\
\hline SLS-2 & 0.710 & 4.057 & 1.745 & yellow-orange & IF & grossular \\
\hline SLS-3 & 0.335 & 4.188 & NR & red-orange & IF & spessartine \\
\hline SLS-4 & 0.550 & 4.074 & NR & orange & IF & spessartine \\
\hline SLS-5 & 0.790 & 4.158 & NR & brown-red & IF & spessartine \\
\hline SLS-6 & 0.470 & 4.273 & NR & orande-red & IF & spessartine \\
\hline SLS-7 & 2.355 & 4.443 & $1.78\left(\mathrm{hm}^{*}\right)$ & dark purple-red & IF & $\begin{array}{l}\text { star al- } \\
\text { mandine }\end{array}$ \\
\hline SLS-8 & 2.170 & 3.875 & $1.78(\mathrm{hm})$ & dark purple-red & IF & $\begin{array}{l}\text { star al- } \\
\text { mandine }\end{array}$ \\
\hline SLS-9 & 1.560 & 4.160 & 1.780 & purple-red & IF & almandine \\
\hline SLS-10 & 1.485 & 4.368 & 1.780 & purple-red & IF & almandine \\
\hline SLS-11 & 1.450 & 3.766 & 1.760 & purple-red & IF & rhodolite \\
\hline SLS-12 & 1.360 & 3.942 & 1.760 & purple-red & IF & rhodolite \\
\hline SLS-13 & 1.000 & 4.082 & 1.765 & brown-red & IF & rhodolite \\
\hline SLS-14 & 1.040 & 3.586 & 1.750 & orande-red & IF & grossular \\
\hline SLS-15 & 0.915 & 3.588 & 1.750 & orande-red & IF & grossular \\
\hline SLS-16 & 0.745 & 3.921 & 1.775 & purple-gray (daylight), & IF & $\operatorname{Ccg} *$ \\
\hline SLS-17 & 0.610 & 4.067 & 1.776 & orange-brown & IF & $\operatorname{Ccg} *$ \\
\hline SLS-18 & 0.750 & 3.947 & 1.773 & (incandescent light) & IF & $\mathrm{Ccg} *$ \\
\hline SLS-19 & 0.490 & 3.630 & 1.738 & green & IF & tsavorite \\
\hline SLS-20 & 0.420 & 3.360 & 1.733 & green & IF & tsavorite \\
\hline SLS-21 & 1.180 & 3.746 & 1.758 & rose-red & IF & $\operatorname{Rrg} *$ \\
\hline SLS-22 & 0.245 & 4.083 & NR & grass green & IF & demantoid \\
\hline SLS-23 & 0.525 & 3.621 & NR & yellow-green & IF & andradite \\
\hline SLS-24 & 0.920 & 3.680 & 1.765 & green-yellow & IF & $\mathrm{Mlg}^{*}$ \\
\hline SLS-25 & 0.875 & 3.646 & 1.767 & green-yellow & IF & Mlg* \\
\hline
\end{tabular}

* Abbreviation: NR = negative reading, $\mathrm{hm}=$ hyperopia method. UVF $=$ Ultraviolet fluorescence, $\mathrm{IF}=$ inert fluorescence. $\mathrm{Ccg}=$ Color-change garnet, $\mathrm{Rrg}=$ Rose-red garnet, Mlg = Mali garnet.

The chemical composition of all samples was tested by laser ablation-inductively coupled plasma-mass spectrometry (LA-ICP-MS, Agilent, Santa Clara, CA, UAS) at a $23.32 \mathrm{kV}$ accelerating voltage performed at the Wuhan Sample Solution Analytical Technology Co. It is a micro-damaging test method and can prevent the destruction of the sample. The ablation spot size was $44 \mu \mathrm{m}$. The testing points were selected at the waist edge or pavilion position of faceted samples or at the bottom position of arc samples. $\mathrm{Fe}^{2+}-\mathrm{Fe}^{3+}$ redistribution of garnet samples from LA-ICP-MS analyses was recalculated using the general equation to estimate $\mathrm{Fe}^{3+}$ [21].

Raman spectroscopy of 21 samples was performed with a Horiba Lab RAM HR Evolution confocal micro-Raman spectrometer coupled with a Leica microscope, using a solid-state Nd-YAG laser at $532 \mathrm{~nm}$ (Horiba Scentific, Paris, France). Raman spectra were collected in the range of $4000-200 \mathrm{~cm}^{-1}$ and used a $6 \mathrm{~mm}$ raster, performed at the State Key Laboratory of Geological Processes and Mineral Resources, China University of Geosciences (Wuhan). Each sample selected a test position without impurities.

Infrared spectroscopy of all samples was performed using a Bruker Vertex 80 Fouriertransform infrared (FTIR) spectrometer (Bruker, Ettlingen, German) at the Gemological Institute, China University of Geosciences (Wuhan). The specular reflection method was used (Kramers-Kronig transformation) under the following conditions: $220 \mathrm{~V}$ scanning voltage, $6 \mathrm{~mm}$ raster, $10 \mathrm{kHz}$ scanning rate, 32 scans, $400-4000 \mathrm{~cm}^{-1}$ range, and $4 \mathrm{~cm}^{-1}$ resolution. The Raman and FTIR spectra were recorded from the randomly oriented specimens. The nonpolarized spectra were shown in this report for the gem-quality garnet samples.

\section{Results}

\subsection{Gem Properties of the Garnet Samples}

Garnet types of the studied samples are primally determined using standard gemological methods. The tested RI and SG values of the samples are listed in Table 1. The four green samples did not appear red through the Chelsea filter. All the garnet samples are inert fluorescence. Their inclusions were not studied in detail because they are not 
usually be used to identify garnet types. In the studied samples, there are two kinds of garnets having special optical effects. One is star garnet. The samples are dark purple and translucence having white six-rayed (SLS-7) and four-rayed (SLS-8) stars, which belong to almandine according to RI and SG values [1,22]. The other is color-changing garnet. The samples (SLS-16 18) are purple-gray in daylight and orange-brown in incandescent light as shown in Figure 1. Rose-red garnet (SLS-21) and Mali garnets (SLS-24 and 25) also have been tested in this study. Sample SLS-22 is demantoid with a grass green color. The gemological properties of the demantoid are consistent with those reported RI and SG values $[11,23]$. The garnet types of some of the studied samples cannot be determined only by gem properties. The colors of the garnet samples are described in Table 1, and the UV-Vis absorption spectra of the samples are shown in Supplementary. The determination of garnet types is checked by the following chemical analysis.

\subsection{Chemical Composition Analysis}

The quantitative chemical compositions were calculated assuming ideal stoichiometry of 8 cations on a 12-oxygen basis and are given in Table 2. The end-member components of the garnet samples are also given in Table 2. Chemical compositional triangular plots were drawn according to the content of the end-members in the pyralspite subgroup and ugrandite subgroup (Figure 2). Most of the samples are nearly located in the boundary of the triangular plots, indicating they are nearly two binary garnet solid solutions.

Table 2. Chemical composition of garnet samples obtained by LA-ICP-MS.

\begin{tabular}{|c|c|c|c|c|c|c|c|c|c|}
\hline $\begin{array}{c}\text { Sample } \\
\text { No. }\end{array}$ & SLS-1 & SLS-2 & SLS-3 & SLS-4 & SSLS-5 & SLS-6 & SSLS-7 & SSLS-8 & SSLS-9 \\
\hline \multicolumn{10}{|c|}{ Oxides(wt.\%) } \\
\hline $\mathrm{SiO}_{2}$ & 36.322 & 39.179 & 36.121 & 36.573 & 36.645 & 36.481 & 38.184 & 38.541 & 37.807 \\
\hline $\mathrm{TiO}_{2}$ & 0.085 & 0.202 & 0.024 & 0.098 & 0.109 & 0.274 & 0.080 & 0.026 & 0.006 \\
\hline $\mathrm{Al}_{2} \mathrm{O}_{3}$ & 19.295 & 21.217 & 19.553 & 19.994 & 19.553 & 19.552 & 20.380 & 20.340 & 20.397 \\
\hline $\mathrm{Cr}_{2} \mathrm{O}_{3}$ & 0 & 0 & 0 & 0 & 0 & 0 & 0 & 0 & 0 \\
\hline $\mathrm{FeO}_{\mathrm{T}}^{\mathrm{a}}$ & 0.348 & 2.352 & 4.554 & 2.100 & 8.557 & 4.939 & 35.152 & 34.010 & 35.777 \\
\hline $\mathrm{MnO}$ & 43.270 & 0.096 & 38.992 & 40.416 & 34.151 & 37.907 & 0.510 & 0.498 & 1.788 \\
\hline $\mathrm{MgO}$ & 0.008 & 0.118 & 0.005 & 0.008 & 0.017 & 0.002 & 3.972 & 3.440 & 2.592 \\
\hline $\mathrm{CaO}$ & 0.178 & 36.713 & 0.285 & 0.396 & 0.404 & 0.263 & 0.400 & 1.914 & 0.434 \\
\hline $\mathrm{V}_{2} \mathrm{O}_{3}$ & 0 & 0 & 0 & 0 & 0 & 0 & 0 & 0 & 0 \\
\hline $\mathrm{Na}_{2} \mathrm{O}$ & 0.005 & 0.002 & 0.005 & 0.005 & 0 & 0.012 & 0.042 & 0.020 & 0.021 \\
\hline $\mathrm{K}_{2} \mathrm{O}$ & 0.001 & 0.001 & 0 & 0.001 & 0.005 & 0 & 0 & 0 & 0.001 \\
\hline $\mathrm{P}_{2} \mathrm{O}_{5}$ & 0.246 & 0.019 & 0.225 & 0.196 & 0.191 & 0.254 & 0.259 & 0.040 & 0.098 \\
\hline Total & 99.758 & 99.899 & 99.764 & 99.787 & 99.632 & 99.684 & 98.979 & 98.829 & 98.921 \\
\hline \multicolumn{10}{|c|}{ Ions based on 12 oxygens } \\
\hline Si & 3.024 & 2.968 & 3.009 & 3.021 & 3.034 & 3.023 & 3.080 & 3.101 & 3.077 \\
\hline $\mathrm{Ti}$ & 0.005 & 0.016 & 0.002 & 0.006 & 0.007 & 0.015 & 0.005 & 0.002 & 0.001 \\
\hline $\mathrm{Al}$ & 1.893 & 1.894 & 1.918 & 1.946 & 1.908 & 1.910 & 1.939 & 1.929 & 1.957 \\
\hline Gr & 0 & 0 & 0 & 0 & 0 & 0 & 0 & 0 & 0 \\
\hline $\mathrm{Fe}^{3+}$ & 0.022 & 0.134 & 0.046 & 0 & 0 & 0 & 0 & 0 & 0 \\
\hline $\mathrm{Fe}^{2+}$ & 0 & 0 & 0.266 & 0.145 & 0.593 & 0.342 & 2.373 & 2.289 & 2.435 \\
\hline $\mathrm{Mn}$ & 3.051 & 0.006 & 2.748 & 2.828 & 2.395 & 2.661 & 0.035 & 0.034 & 0.123 \\
\hline $\mathrm{Mg}$ & 0.001 & 0.013 & 0.001 & 0.001 & 0.002 & 0.001 & 0.478 & 0.413 & 0.315 \\
\hline $\mathrm{Ca}$ & 0.016 & 2.980 & 0.025 & 0.035 & 0.036 & 0.0234 & 0.035 & 0.165 & 0.038 \\
\hline $\mathrm{V}$ & 0 & 0 & 0 & 0 & 0 & 0 & 0 & 0 & 0 \\
\hline
\end{tabular}


Table 2. Cont.

\begin{tabular}{|c|c|c|c|c|c|c|c|c|c|}
\hline \multicolumn{10}{|c|}{ Mol\% of end members } \\
\hline Uvarovite & 0 & 0 & 0 & 0 & 0 & 0 & 0 & 0 & 0 \\
\hline Andradite & 1.07 & 6.61 & 2.27 & 1.80 & 3.31 & 3.29 & 0.25 & 0.14 & 0 \\
\hline Pyrope & 0.03 & 0.44 & 0.02 & 0.03 & 0.07 & 0.01 & 16.39 & 14.24 & 10.72 \\
\hline Spessartine & 99.45 & 0.20 & 90.40 & 95.11 & 80.91 & 89.84 & 1.20 & 1.17 & 4.20 \\
\hline Grossular & 0 & 91.33 & 0 & 0 & 0 & 0 & 0.94 & 5.55 & 1.29 \\
\hline Almandine & 0 & 0 & 8.74 & 3.68 & 17.81 & 9.37 & 81.22 & 78.89 & 82.98 \\
\hline Other & 0 & 1.42 & 0 & 0 & 0 & 0 & 0 & 0 & 0.82 \\
\hline $\begin{array}{c}\text { Sample } \\
\text { No. }\end{array}$ & SLS-10 & SLS-11 & SLS-12 & SLS-13 & SLS-14 & SLS-15 & SLS-16 & SLS-17 & SLS-18 \\
\hline \multicolumn{10}{|c|}{ Oxides(wt.\%) } \\
\hline $\mathrm{SiO}_{2}$ & 38.179 & 41.087 & 41.203 & 41.108 & 39.475 & 38.812 & 38.789 & 39.593 & 39.153 \\
\hline $\mathrm{TiO}_{2}$ & 0.017 & 0.016 & 0.013 & 0.014 & 0.163 & 0.199 & 0.076 & 0.043 & 0.105 \\
\hline $\mathrm{Al}_{2} \mathrm{O}_{3}$ & 20.561 & 22.531 & 22.286 & 21.660 & 19.411 & 19.433 & 20.529 & 20.705 & 20.620 \\
\hline $\mathrm{Cr}_{2} \mathrm{O}_{3}$ & 0 & 0 & 0 & 0 & 0 & 0 & 0 & 0.093 & 0.106 \\
\hline $\mathrm{FeO}_{\mathrm{T}}{ }^{\mathrm{a}}$ & 35.334 & 17.622 & 18.090 & 20.594 & 4.000 & 4.575 & 1.987 & 1.892 & 2.498 \\
\hline $\mathrm{MnO}$ & 1.445 & 0.487 & 0.586 & 0.767 & 0.257 & 0.261 & 29.000 & 27.407 & 27.401 \\
\hline $\mathrm{MgO}$ & 2.761 & 13.698 & 13.357 & 11.819 & 0.141 & 0.375 & 7.073 & 8.200 & 7.964 \\
\hline $\mathrm{CaO}$ & 0.469 & 3.960 & 3.818 & 3.357 & 36.400 & 36.169 & 1.809 & 1.813 & 1.465 \\
\hline $\mathrm{V}_{2} \mathrm{O}_{3}$ & 0 & 0 & 0 & 0 & 0 & 0 & 0 & 0 & 0 \\
\hline $\mathrm{Na}_{2} \mathrm{O}$ & 0.029 & 0.005 & 0.004 & 0.006 & 0.003 & 0.004 & 0.020 & 0.011 & 0.014 \\
\hline $\mathrm{K}_{2} \mathrm{O}$ & 0 & 0.001 & 0.001 & 0 & 0 & 0.001 & 0 & 0.001 & 0.001 \\
\hline $\mathrm{P}_{2} \mathrm{O}_{5}$ & 0.145 & 0.050 & 0.057 & 0.052 & 0.013 & 0.020 & 0.055 & 0.061 & 0.087 \\
\hline Total & 98.940 & 99.457 & 99.415 & 99.377 & 99.863 & 99.849 & 99.338 & 99.819 & 99.414 \\
\hline \multicolumn{10}{|c|}{ Ions based on 12 oxygens } \\
\hline $\mathrm{Si}$ & 3.094 & 3.046 & 3.062 & 3.087 & 3.009 & 2.967 & 3.039 & 3.065 & 3.047 \\
\hline $\mathrm{Ti}$ & 0.001 & 0.001 & 0.001 & 0.001 & 0.009 & 0.011 & 0.005 & 0.003 & 0.006 \\
\hline $\mathrm{Al}$ & 1.964 & 1.969 & 1.952 & 1.917 & 1.744 & 1.751 & 1.896 & 1.889 & 1.891 \\
\hline $\mathrm{Gr}$ & 0 & 0 & 0 & 0 & 0 & 0 & 0 & 0.006 & 0.007 \\
\hline $\mathrm{Fe}^{3+}$ & 0 & 0 & 0 & 0 & 0.229 & 0.263 & 0 & 0 & 0 \\
\hline $\mathrm{Fe}^{2+}$ & 2.395 & 1.093 & 1.124 & 1.294 & 0 & 0 & 0.130 & 0.123 & 0.163 \\
\hline $\mathrm{Mn}$ & 0.099 & 0.031 & 0.037 & 0.049 & 0.017 & 0.017 & 1.924 & 1.774 & 1.806 \\
\hline $\mathrm{Mg}$ & 0.328 & 1.514 & 1.480 & 1.323 & 0.016 & 0.043 & 0.826 & 0.946 & 0.924 \\
\hline $\mathrm{Ca}$ & 0.041 & 0.315 & 0.304 & 0.270 & 2.972 & 2.963 & 0.152 & 0.150 & 0.122 \\
\hline $\mathrm{V}$ & 0 & 0 & 0 & 0 & 0 & 0 & 0 & 0 & 0 \\
\hline \multicolumn{10}{|c|}{$\mathrm{Mol} \%$ of end members } \\
\hline Uvarovite & 0 & 0 & 0 & 0 & 0 & 0 & 0 & 0.29 & 0.33 \\
\hline Andradite & 0 & 0 & 0 & 0 & 11.45 & 13.06 & 3.84 & 3.08 & 3.42 \\
\hline Pyrope & 11.14 & 51.27 & 50.71 & 46.68 & 0.53 & 1.41 & 27.94 & 32.27 & 31.35 \\
\hline Spessartine & 3.37 & 1.04 & 1.26 & 1.68 & 0.55 & 0.56 & 65.08 & 60.48 & 61.28 \\
\hline Grossular & 1.38 & 10.65 & 10.00 & 8.04 & 87.46 & 84.96 & 1.30 & 1.76 & 0.39 \\
\hline Almandine & 81.30 & 37.00 & 38.03 & 43.60 & 0 & 0 & 1.84 & 2.12 & 3.23 \\
\hline Other & 2.82 & 0.05 & 0 & 0 & 0 & 0 & 0 & 0 & 0 \\
\hline $\begin{array}{c}\text { Sample } \\
\text { No. }\end{array}$ & SLS-19 & SLS-20 & SLS-21 & SLS-22 & \multicolumn{2}{|c|}{ SLS-23 } & \multicolumn{2}{|c|}{ SLS-24 } & SLS-25 \\
\hline \multicolumn{10}{|c|}{ Oxides(wt.\%) } \\
\hline $\mathrm{SiO}_{2}$ & 39.072 & 38.936 & 41.258 & 34.982 & \multicolumn{2}{|c|}{34.678} & \multicolumn{2}{|c|}{38.766} & 38.654 \\
\hline $\mathrm{TiO}_{2}$ & 0.354 & 0.538 & 0.029 & 0.041 & \multicolumn{2}{|c|}{0} & \multicolumn{2}{|c|}{0.243} & 0.432 \\
\hline $\mathrm{Al}_{2} \mathrm{O}_{3}$ & 21.719 & 21.833 & 21.826 & 0.072 & \multicolumn{2}{|c|}{0.002} & \multicolumn{2}{|c|}{17.419} & 16.788 \\
\hline $\mathrm{Cr}_{2} \mathrm{O}_{3}$ & 0 & 0 & 0 & 0.296 & \multicolumn{2}{|c|}{0} & \multicolumn{2}{|c|}{0} & 0 \\
\hline $\mathrm{FeO}_{\mathrm{T}}^{\mathrm{a}}$ & 0.073 & 0.072 & 19.880 & 29.054 & \multicolumn{2}{|c|}{29.629} & \multicolumn{2}{|c|}{6.504} & 7.158 \\
\hline $\mathrm{MnO}$ & 1.328 & 1.019 & 0.711 & 0.010 & & & & & 0.102 \\
\hline $\mathrm{MgO}$ & 2.535 & 0.561 & 13.960 & 0.094 & & & & & 0.471 \\
\hline $\mathrm{CaO}$ & 36.275 & 36.579 & 1.427 & 34.449 & & & & & 36.139 \\
\hline $\mathrm{V}_{2} \mathrm{O}_{3}$ & 0.294 & 0.201 & 0 & 0 & & & & & 0 \\
\hline $\mathrm{Na}_{2} \mathrm{O}$ & 0.004 & 0.004 & 0.020 & 0.001 & & & & & 0.002 \\
\hline $\mathrm{K}_{2} \mathrm{O}$ & 0 & 0 & 0.001 & 0 & & & & & 0.001 \\
\hline
\end{tabular}


Table 2. Cont.

\begin{tabular}{|c|c|c|c|c|c|c|c|}
\hline $\mathrm{P}_{2} \mathrm{O}_{5}$ & 0.017 & 0.014 & 0.176 & 0.010 & 0.013 & 0.022 & 0.018 \\
\hline Total & 101.671 & 99.757 & 99.288 & 99.009 & 99.171 & 99.780 & 99.765 \\
\hline \multicolumn{8}{|c|}{ Ions based on 12 oxygens } \\
\hline $\mathrm{Si}$ & 2.969 & 2.953 & 3.077 & 2.991 & 2.968 & 2.973 & 2.971 \\
\hline $\mathrm{Ti}$ & 0.020 & 0.031 & 0.002 & 0.003 & 0 & 0.014 & 0.025 \\
\hline $\mathrm{Al}$ & 1.945 & 1.951 & 1.918 & 0.007 & 0 & 1.574 & 1.521 \\
\hline $\mathrm{Gr}$ & 0 & 0 & 0 & 0.020 & 0 & 0 & 0 \\
\hline $\mathrm{Fe}^{3+}$ & 0.004 & 0.004 & 0 & 1.869 & 1.909 & 0.417 & 0.460 \\
\hline $\mathrm{Fe}^{2+}$ & 0 & 0 & 1.240 & 0 & 0 & 0 & 0 \\
\hline Mn & 0.086 & 0.066 & 0.045 & 0.001 & 0.001 & 0.010 & 0.007 \\
\hline $\mathrm{Mg}$ & 0.061 & 0.063 & 1.552 & 0.012 & 0.016 & 0.062 & 0.054 \\
\hline $\mathrm{Ca}$ & 2.953 & 2.972 & 0.114 & 3.156 & 3.184 & 2.968 & 2.976 \\
\hline $\mathrm{V}$ & 0.034 & 0.024 & 0 & 0 & 0 & 0 & 0 \\
\hline \multicolumn{8}{|c|}{ Mol \% of end-members } \\
\hline Uvarovite & 0 & 0 & 0 & 0.95 & 0 & 0 & 0 \\
\hline Andradite & 0.20 & 0.20 & 0 & 88.50 & 89.45 & 20.58 & 22.73 \\
\hline Pyrope & 1.96 & 2.05 & 53.12 & 0.38 & 0.49 & 2.05 & 1.78 \\
\hline Spessartine & 2.76 & 2.11 & 1.54 & 0.02 & 0.01 & 0.34 & 0.22 \\
\hline Grossular & 95.09 & 95.65 & 2.40 & 10.16 & 10.05 & 77.04 & 75.28 \\
\hline Almandine & 0 & 0 & 42.95 & 0 & 0 & 0 & 0 \\
\hline Other & 0 & 0 & 0 & 0 & 0 & 0 & 0 \\
\hline
\end{tabular}

a $\mathrm{T}=$ Total

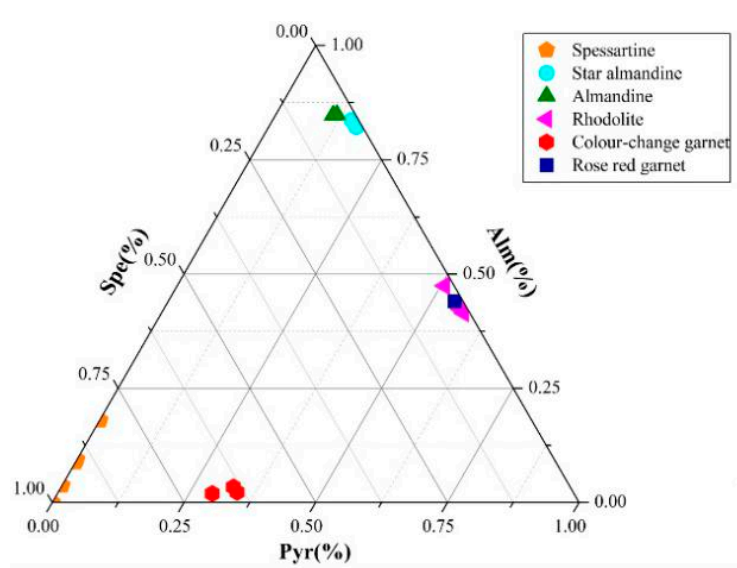

a

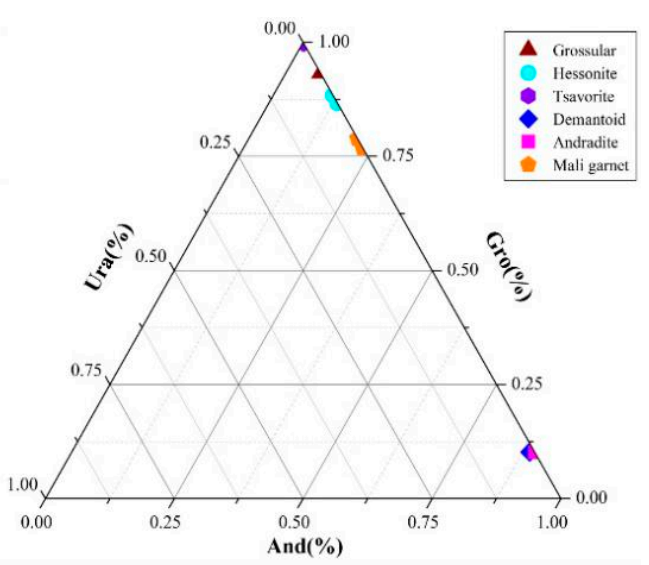

$\mathrm{b}$

Figure 2. The chemical compositional triangular plot of gem garnet samples. (a) The Pyropespessartine-almandine triangular plot of the sixteen samples belonging to the pyralspite subgroup. (b) The andradite-grossular-uvarovite triangular plot of the nine samples belonging to the ugrandite subgroup. Chemical composition details see Table 2.

Samples SLS 11 13 examined are the intermediate members of the pyrope-almandine series with typical color and gem properties of rhodolite [1]. The rose-red garnet (SLS-21) has a special rose-red color with compositions of approximately $\mathrm{Py}_{53} \mathrm{Al}_{43} \mathrm{Gr}_{2} \mathrm{Sp}_{2}$. However, according to $\mathrm{RIM}$ values $\left(\mathrm{N}\left(\mathrm{Fe}^{2+}\right) / \mathrm{N}\left(\mathrm{Fe}^{2+}+\mathrm{Mg}^{2+}\right)\right)$, the studied rhodolite and rose-red garnet samples belong to pyrope specie $(\mathrm{RIM}<0.5)$ [24]. The studied almandine samples (SLS-7 10) contain a different amount of $\mathrm{Mg}^{2+}$ substituting $\mathrm{Fe}^{2+}$ in A site, and contain a little amount of spessartine and a grossular component.

There are three kinds of chemical substitution series in the studied spessartine species, near ideal end-member spessartine (SLS-1), spessartine-almandine series (SLS-3 6), and spessartine-pyrope series (SLS-16 18). The studied color-changing garnets belong to the spessartine-pyrope series. The color-changing phenomenon may be attributable to a little amount of $\mathrm{Cr}_{2} \mathrm{O}_{3}$ [12]. However, no chromium was detected in sample SLS-16. The color-changing causes will not be discussed here. 
The grossular species of the studied samples contain three varieties, tsavorite, hessonite, and Mali garnet. Tsavorite samples (SLS-19 and 20) are near the ideal end-member grossular composition (above 95\% wt.\% grossular), having a very little amount of iron cations in the structure. Their minor $\mathrm{V}_{2} \mathrm{O}_{3}$ contents cause a green hue. $\mathrm{No}_{\mathrm{Cr}} \mathrm{O}_{3}$ was detected in the samples, which has no contribution to the color [11]. Hessonite is a variety of grossular that contains sufficient $\mathrm{Fe}^{3+}$ to cause the orange-yellow color [25]. Sample SLS-2 appears lighter orange-yellow color than sample SLS-14 and SLS-15 because of fewer $\mathrm{Fe}^{3+}$ cations. Two Mali garnets are greenish-yellow (SLS-24) and yellow (SLS-25) with compositions of $\mathrm{Gr}_{77} \mathrm{And}_{21} \mathrm{Py}_{2}$ and $\mathrm{Gr}_{75} \mathrm{And}_{23} \mathrm{Py}_{2}$, respectively [10].

Two andradite samples (SLS-22 and 23) are representative and have dominated $\mathrm{Fe}^{3+}$ cations in the B site. They have similar andradite (88.50 wt.\% and $89.45 \mathrm{wt} . \%$ andradite, respectively) and grossular (10.16 wt.\% and $10.05 \mathrm{wt} . \%$ grossular, respectively) components but different green colors. One of them (SLS-22) is demantoid, which is grass green, with minor chromium (nearly $0.3 \%$ wt. $\% \mathrm{Cr}_{2} \mathrm{O}_{3}$ ) contributing the valued green color [23]. The other andradite (SLS-23) is yellow-green without the Cr component.

In order to obviously show the relationship between cations and spectra in garnet types, the calculated chemical structure formula of the studied garnets was listed in Table 3. The samples in Table 3 were classified into two groups according to the dominant cations in the B site. The aluminosilicate garnet, including pyrope, almandine, spessartine, and grossular, has $\mathrm{Al}^{3+}$ in the $\mathrm{B}$ site but has different divalent cations in the $\mathrm{A}$ site. The divalent cations have different masses and radius. However, andradite has relatively larger cations both in the A site $\left(\mathrm{Ca}^{2+}\right)$ and in the $\mathrm{B}$ site $\left(\mathrm{Fe}^{3+}\right)$ than aluminosilicate garnet has. So, andradite is expected to show more different Raman and IR spectra than other garnet species. In Table 3, the order of the samples of the same types is listed according to the increasing order of the main garnet component in the aluminosilicate garnets.

Table 3. List of chemical structure formulas of all the studied garnets.

\begin{tabular}{|c|c|c|c|}
\hline Classifications & Species & Sample No. & chemical Structure Formula \\
\hline \multirow{4}{*}{$\mathrm{B}^{3+}=\mathrm{Al}^{3+}$} & Pyrope & $\begin{array}{l}\text { SLS-13 } \\
\text { SLS-12 } \\
\text { SLS-11 } \\
\text { SLS-21 }\end{array}$ & $\begin{array}{l}\left(\mathrm{Mg}_{1.32} \mathrm{Fe}^{2+}{ }_{1.29} \mathrm{Ca}_{0.27} \mathrm{Mn}_{0.05}\right)_{2.93} \mathrm{Al}_{1.92}\left[\mathrm{Si}_{3.19} \mathrm{O}_{12}\right] \\
\left(\mathrm{Mg}_{1.48} \mathrm{Fe}^{2+}{ }_{1.12} \mathrm{Ca}_{0.30} \mathrm{Mn}_{0.04}\right)_{2.94} \mathrm{Al}_{1.95}\left[\mathrm{Si}_{3.06} \mathrm{O}_{12}\right] \\
\left(\mathrm{Mg}_{1.51} \mathrm{Fe}^{2+}{ }_{1.09} \mathrm{Ca}_{0.31} \mathrm{Mn}_{0.03}\right)_{2.94} \mathrm{Al}_{1.97}\left[\mathrm{Si}_{3.05} \mathrm{O}_{12}\right] \\
\left(\mathrm{Mg}_{1.55} \mathrm{Fe}^{2+}{ }_{1.24} \mathrm{Ca}_{0.11} \mathrm{Mn}_{0.04}\right)_{2.94} \mathrm{Al}_{1.92}\left[\mathrm{Si}_{3.08} \mathrm{O}_{12}\right]\end{array}$ \\
\hline & Almandine & $\begin{array}{l}\text { SLS-8 } \\
\text { SLS-7 } \\
\text { SLS-10 } \\
\text { SLS-9 }\end{array}$ & $\begin{array}{l}\left(\mathrm{Fe}^{2+}{ }_{2.29} \mathrm{Mg}_{0.41} \mathrm{Ca}_{0.17} \mathrm{Mn}_{0.03}\right)_{2.90} \mathrm{Al}_{1.93}\left[\mathrm{Si}_{3.10} \mathrm{O}_{12}\right] \\
\left(\mathrm{Fe}^{2+}{ }_{2.37} \mathrm{Mg}_{0.48} \mathrm{Mn}_{0.03} \mathrm{Ca}_{0.04}\right)_{2.92} \mathrm{Al}_{1.94}\left[\mathrm{Si}_{3.08} \mathrm{O}_{12}\right] \\
\left(\mathrm{Fe}^{2+}{ }_{2.40} \mathrm{Mg}_{0.33} \mathrm{Mn}_{0.10} \mathrm{Ca}_{0.04}\right)_{2.87} \mathrm{Al}_{1.96}\left[\mathrm{Si}_{3.09} \mathrm{O}_{12}\right] \\
\left(\mathrm{Fe}^{2+}{ }_{2.44} \mathrm{Mg}_{0.31} \mathrm{Mn}_{0.12} \mathrm{Ca}_{0.04}\right)_{2.91} \mathrm{Al}_{1.96}\left[\mathrm{Si}_{3.08} \mathrm{O}_{12}\right]\end{array}$ \\
\hline & Spessartine & $\begin{array}{l}\text { SLS-17 } \\
\text { SLS-18 } \\
\text { SLS-16 } \\
\text { SLS-5 } \\
\text { SLS-6 } \\
\text { SLS-3 } \\
\text { SLS-4 } \\
\text { SLS-1 }\end{array}$ & $\begin{array}{c}\left(\mathrm{Mn}_{1.77} \mathrm{Mg}_{0.95} \mathrm{Ca}_{0.15} \mathrm{Fe}^{2+}{ }_{0.12}\right)_{2.99}\left(\mathrm{Al}_{1.89} \mathrm{Cr}_{0.01}\right)_{1.90}\left[\mathrm{Si}_{3.07} \mathrm{O}_{12}\right] \\
\left(\mathrm{Mn}_{1.81} \mathrm{Mg}_{0.92} \mathrm{Fe}^{2+}{ }_{0.16} \mathrm{Ca}_{0.12}\right)_{3.01}\left(\mathrm{Al}_{1.89} \mathrm{Cr}_{0.01} \mathrm{Ti}_{0.01}\right)_{1.91}\left[\mathrm{Si}_{3.05} \mathrm{O}_{12}\right] \\
\left(\mathrm{Mn}_{1.92} \mathrm{Mg}_{0.83} \mathrm{Ca}_{0.15} \mathrm{Fe}^{2+}{ }_{0.13}\right)_{3.03} \mathrm{Al}_{1.90}\left[\mathrm{Si}_{3.04} \mathrm{O}_{12}\right] \\
\left(\mathrm{Mn}_{2.39} \mathrm{Fe}^{2+}{ }_{0.59} \mathrm{Ca}_{0.04}\right)_{3.02}\left(\mathrm{Al}_{1.91} \mathrm{Ti}_{0.01}\right)_{1.92}\left[\mathrm{Si}_{3.04} \mathrm{O}_{12}\right] \\
\left(\mathrm{Mn}_{2.66} \mathrm{Fe}^{2+}{ }_{0.34} \mathrm{Ca}_{0.02}\right)_{3.02}\left(\mathrm{Al}_{1.91} \mathrm{Ti}_{0.02}\right)_{1.93}\left[\mathrm{Si}_{3.02} \mathrm{O}_{12}\right] \\
\left(\mathrm{Mn}_{2.75} \mathrm{Fe}^{2+}{ }_{0.27} \mathrm{Ca}_{0.03}\right)_{3.05}\left(\mathrm{Al}_{1.92} \mathrm{Fe}^{3+}{ }_{0.05}\right)_{1.97}\left[\mathrm{Si}_{3.01} \mathrm{O}_{12}\right] \\
\left(\mathrm{Mn}_{2.83} \mathrm{Fe}^{2+}{ }_{0.15} \mathrm{Ca}_{0.04}\right)_{3.02}\left(\mathrm{Al}_{1.95} \mathrm{Ti}_{0.01}\right)_{1.96}\left[\mathrm{Si}_{3.02} \mathrm{O}_{12}\right] \\
\left(\mathrm{Mn}_{3.05} \mathrm{Ca}_{0.02}\right)_{3.07}\left(\mathrm{Al}_{1.89} \mathrm{Fe}^{3+}{ }_{0.02} \mathrm{Ti}_{0.01}\right)_{1.92}\left[\mathrm{Si}_{3.02} \mathrm{O}_{12}\right]\end{array}$ \\
\hline & Grossular & $\begin{array}{l}\text { SLS-25 } \\
\text { SLS-24 } \\
\text { SLS-15 } \\
\text { SLS-14 } \\
\text { SLS-2 } \\
\text { SLS-19 } \\
\text { SLS-20 }\end{array}$ & $\begin{array}{c}\left(\mathrm{Ca}_{2.98} \mathrm{Mg}_{0.05} \mathrm{Mn}_{0.01}\right)_{3.04}\left(\mathrm{Al}_{1.52} \mathrm{Fe}^{3+}{ }^{0.46} \mathrm{Ti}_{0.03}\right)_{2.01}\left[\mathrm{Si}_{2.97} \mathrm{O}_{12}\right] \\
\left(\mathrm{Ca}_{2.97} \mathrm{Mg}_{0.06} \mathrm{Mn}_{0.01}\right)_{3.04}\left(\mathrm{Al}_{1.57} \mathrm{Fe}^{3+}{ }^{0.42} \mathrm{Ti}_{0.01}\right)_{2.00}\left[\mathrm{Si}_{2.97} \mathrm{O}_{12}\right] \\
\left(\mathrm{Ca}_{2.96} \mathrm{Mg}_{0.04} \mathrm{Mn}_{0.02}\right)_{3.02}\left(\mathrm{Al}_{1.75} \mathrm{Fe}^{3+}{ }^{0.26} \mathrm{Ti}_{0.01}\right)_{2.02}\left[\mathrm{Si}_{2.97} \mathrm{O}_{12}\right] \\
\left(\mathrm{Ca}_{2.97} \mathrm{Mn}_{0.02} \mathrm{Mg}_{0.02}\right)_{3.01}\left(\mathrm{Al}_{1.74} \mathrm{Fe}^{3+}{ }^{0.23} \mathrm{Ti}_{0.01}\right)_{1.98}\left[\mathrm{Si}_{3.01} \mathrm{O}_{12}\right] \\
\left(\mathrm{Ca}_{2.98} \mathrm{Mg}_{0.01} \mathrm{Mn}_{0.01}\right)_{3.00}\left(\mathrm{Al}_{1.89} \mathrm{Fe}^{3+}{ }^{0.13} \mathrm{Ti}_{0.01}\right)_{2.03}\left[\mathrm{Si}_{2.97} \mathrm{O}_{12}\right] \\
\left(\mathrm{Ca}_{2.95} \mathrm{Mn}_{0.09} \mathrm{Mg}_{0.06}\right)_{3.10}\left(\mathrm{Al}_{1.94} \mathrm{~V}_{0.03} \mathrm{Ti}_{0.02}\right)_{1.99}\left[\mathrm{Si}_{2.97} \mathrm{O}_{12}\right] \\
\left(\mathrm{Ca}_{2.97} \mathrm{Mn}_{0.07} \mathrm{Mg}_{0.06}\right)_{3.10}\left(\mathrm{Al}_{1.95} \mathrm{Ti}_{0.03} \mathrm{~V}_{0.02}\right)_{2.00}\left[\mathrm{Si}_{2.95} \mathrm{O}_{12}\right]\end{array}$ \\
\hline $\mathrm{B}^{3+}=\mathrm{Fe}^{3+}$ & Andradite & $\begin{array}{l}\text { SLS-22 } \\
\text { SLS-23 }\end{array}$ & $\begin{array}{c}\left(\mathrm{Ca}_{3.16} \mathrm{Mg}_{0.01}\right)_{3.17}\left(\mathrm{Fe}^{3+}{ }_{1.87} \mathrm{Cr}_{0.02} \mathrm{Al}_{0.01}\right)_{1.90}\left[\mathrm{Si}_{2.99} \mathrm{O}_{12}\right] \\
\left(\mathrm{Ca}_{3.18} \mathrm{Mg}_{0.02}\right)_{3.20} \mathrm{Fe}^{3+}{ }_{1.91}\left[\mathrm{Si}_{2.97} \mathrm{O}_{12}\right]\end{array}$ \\
\hline
\end{tabular}




\subsection{Raman Spectroscopy Features}

According to theoretical factor group analysis on garnet, the total number of vibrations and the number of Raman and infrared active modes were calculated [26]. The total irreducible representation at the $\Gamma$-point is given as follows:

$$
\Gamma=3 \mathrm{~A}_{1 \mathrm{~g}}+5 \mathrm{~A}_{2 \mathrm{~g}}+8 \mathrm{E}_{\mathrm{g}}+14 \mathrm{~F}_{1 \mathrm{~g}}+14 \mathrm{~F}_{2 \mathrm{~g}}+5 \mathrm{~A}_{1 \mathrm{u}}+5 \mathrm{~A}_{2 \mathrm{u}}+10 \mathrm{E}_{\mathrm{u}}+17 \mathrm{~F}_{1 \mathrm{u}}+16 \mathrm{~F}_{2 \mathrm{u}}
$$

A total of 25 modes are Raman active, which are $\mathrm{A}_{1 \mathrm{~g}-}, \mathrm{E}_{\mathrm{g}-}$, and $\mathrm{F}_{2 \mathrm{~g}-}$ modes, and $17 \mathrm{~F}_{1 \mathrm{u}}$ modes are active in the infrared [16].

Raman spectra of the studied samples are shown in Figure 3, and the observed Raman vibrational modes of the samples are described in Table 4 . The zone-center Raman active vibrational modes are considered to be relative to the $\mathrm{SiO}_{4}$-tetrahedra and $\mathrm{A}$ site cations in garnet structure. The B site cations do not produce Raman active vibrations [16].

The range of Raman spectra of garnets can be grouped in five regions: the highest frequency modes $\left(1100 \sim 850 \mathrm{~cm}^{-1}\right)$ are dominated by stretching motions of $\mathrm{Si}-\mathrm{O}\left(\mathrm{Si}-\mathrm{O}_{\text {str }}\right)$, the frequency modes at around $550 \mathrm{~cm}^{-1}$ are dominated by bending motions of $\mathrm{Si}-\mathrm{O}$ ( $\left.\mathrm{Si}-\mathrm{O}_{\text {bend }}\right)$, the frequency modes at around $420 \mathrm{~cm}^{-1}$ are dominated by rotation motions of $\mathrm{SiO}_{4}\left(\mathrm{R}\left(\mathrm{SiO}_{4}\right)^{4-}\right)$, and the modes below $300 \mathrm{~cm}^{-1}$ are due to translational motions of $\mathrm{SiO}_{4}$ $\left(\mathrm{T}\left(\mathrm{SiO}_{4}\right)^{4-}\right)$ and $\mathrm{A}^{2+}(\mathrm{x}, \mathrm{y})$-translations $\left(\mathrm{T}\left(\mathrm{A}^{2+}\right)\right)[16,18]$.

The nonpolarized spectra of the aluminosilicate garnets show similar patterns of $\mathrm{Si}-\mathrm{O}_{\text {str }}$ and $\mathrm{Si}-\mathrm{O}_{\text {bend }}$ modes (Figure $3 \mathrm{a}-\mathrm{d}$ ). However, the frequencies of these modes are different in different garnet species (Table 4), which will be discussed in detail below. The weak $\mathrm{Si}-\mathrm{O}_{\text {str }} \mathrm{E}_{\mathrm{g}}$ mode of all the tested aluminosilicate garnets was not detected herein, but the mode of some garnet species can be observed by polarized spectra when detecting perpendicular to the special crystal plane of the garnet single crystals [16,27]. Tsavorite samples (SLS-19 and 20) have strong fluorescence background and only show weak $\mathrm{Si}-\mathrm{O}_{\text {str }}$ $\mathrm{A}_{1 \mathrm{~g}}$ and $\mathrm{R}\left(\mathrm{SiO}_{4}\right)^{4-} \mathrm{Eg}$ modes. So, they show different Raman spectra in the grossular group, which may result from minor $\mathrm{V}_{2} \mathrm{O}_{3}$ contents in the crystals.

The patterns and frequencies of $\mathrm{Si}-\mathrm{O}_{\text {str }}$ and $\mathrm{Si}-\mathrm{O}_{\text {bend }}$ modes of the studied andradite samples are obviously different from those of the aluminosilicate garnets (Figure 3). The highest frequency of $\mathrm{Si}-\mathrm{O}_{\mathrm{str}} \mathrm{F}_{2 \mathrm{~g}}$ of andradite is lower than $1000 \mathrm{~cm}^{-1}$, while those of the aluminosilicate garnets are higher than $1000 \mathrm{~cm}^{-1}$. The $\mathrm{Si}-\mathrm{O}_{\mathrm{str}} \mathrm{A}_{1 \mathrm{~g}}$ mode of andradite was not detected, while $\mathrm{Si}-\mathrm{O}_{\mathrm{str}} \mathrm{E}_{\mathrm{g}}$ mode $\left(870 \mathrm{~cm}^{-1}\right)$ existed. These phenomena are the same as the reported polarized Raman spectra $[16,27]$.

As shown in Figure 3 the patterns and frequencies of modes below $300 \mathrm{~cm}^{-1}\left[\mathrm{~T}\left(\mathrm{SiO}_{4}\right)^{4-}\right.$ and $\mathrm{T}\left(\mathrm{A}^{2+}\right)$ modes] have much difference among the five garnet species, which could be used to discriminate between the garnet species' minerals. However, because it is still difficult to describe the external vibrations as originating from certain atoms or polyhedral units [18], low-frequency modes are not suitable to establish correlations among chemical composition, structure, and Raman spectra. So, they are not described in detail and discussed herein 



Raman shift $\left(\mathrm{cm}^{-1}\right)$
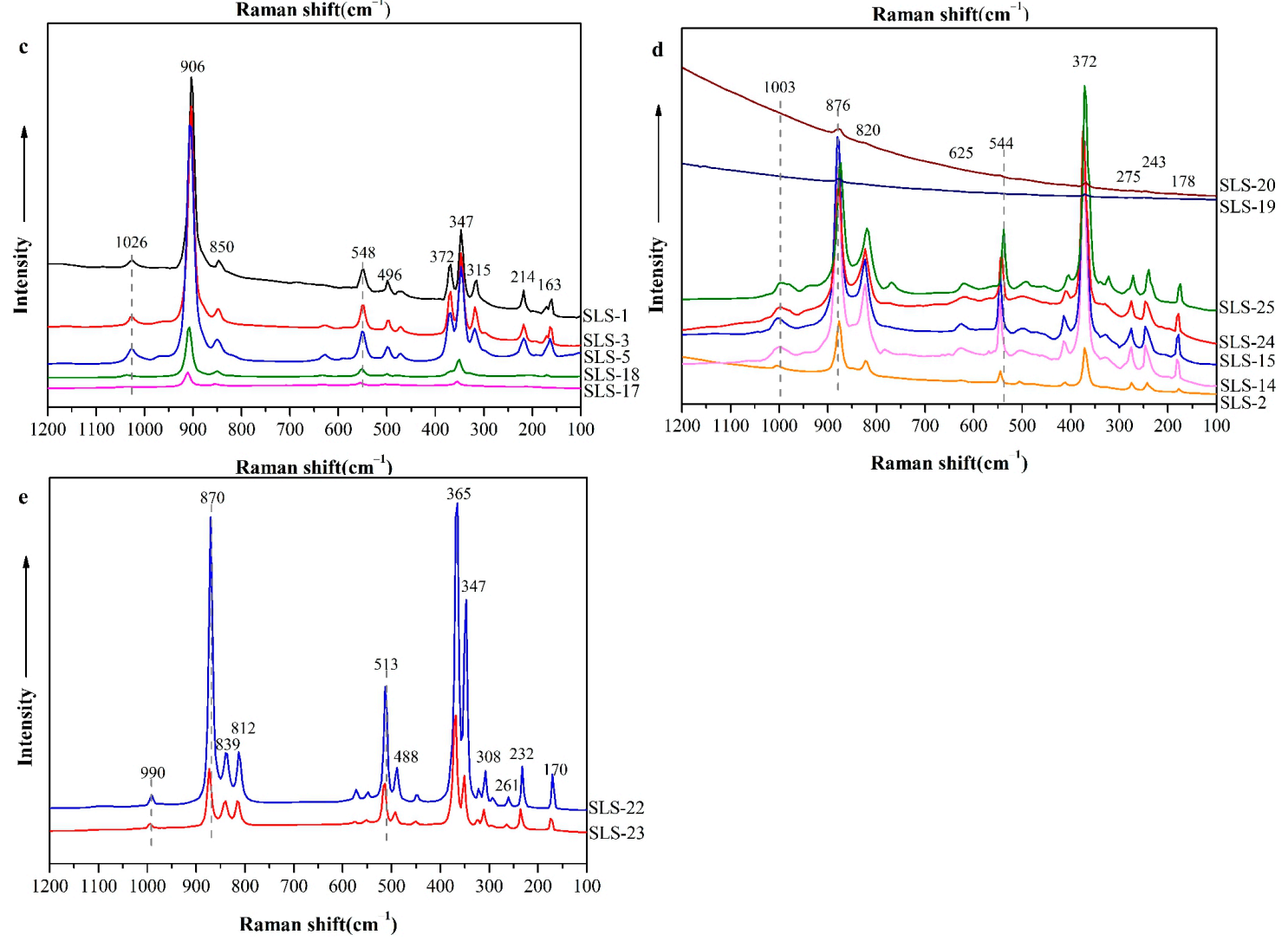

Raman shift(cm ')

Figure 3. The Raman spectra of gem garnet samples. (a) pyrope, (b) almandine, (c) spessartine, (d) grossular, and (e) andradite. 
Table 4. Raman activity and assignment of the studied garnets $\left(\mathrm{cm}^{-1}\right)$.

\begin{tabular}{|c|c|c|c|c|c|c|c|c|c|c|c|c|c|}
\hline \multirow[t]{2}{*}{ Species } & \multicolumn{3}{|c|}{ Si-O Stretching Vibration } & \multicolumn{3}{|c|}{ Si-O Bending Vibration } & \multicolumn{3}{|c|}{$\begin{array}{c}{\left[\mathrm{SiO}_{4}\right]^{4-} \text { Rotational }} \\
\text { Vibration }\end{array}$} & \multicolumn{2}{|c|}{$\begin{array}{c}\left.\mathrm{SiO}_{4}\right]^{4-} \\
\text { Translation } \\
\text { Vibration }\end{array}$} & \multicolumn{2}{|c|}{$\begin{array}{c}\mathrm{A}^{2+} \text { Translation } \\
\text { Vibration }\end{array}$} \\
\hline & $F_{2 g}$ & $E_{g}$ & $\mathbf{A}_{1 \mathrm{~g}}$ & $F_{2 g}$ & $E_{g}$ & $\mathbf{A}_{1 \mathrm{~g}}$ & $F_{2 g}$ & $\mathrm{E}_{\mathrm{g}}$ & $\mathbf{A}_{1 \mathrm{~g}}$ & $F_{2 g}$ & $\mathrm{E}_{\mathrm{g}}$ & $F_{2 g}$ & $E_{g}$ \\
\hline Pyrope & $\begin{array}{c}1046 \\
860\end{array}$ & & 913 & $\begin{array}{l}635 \\
503 \\
485\end{array}$ & & 555 & 317 & & 354 & & 204 & & \\
\hline Almandine & $\begin{array}{l}1039 \\
863\end{array}$ & & 916 & $\begin{array}{l}631 \\
583 \\
500 \\
478\end{array}$ & $\begin{array}{l}596 \\
372\end{array}$ & 555 & & 315 & 347 & & 167 & 211 & \\
\hline Spessartine & $\begin{array}{l}1026, \\
850\end{array}$ & & 906 & $\begin{array}{l}628 \\
496 \\
471\end{array}$ & $\begin{array}{l}590 \\
369\end{array}$ & 548 & & 318 & 347 & & 163 & 214 & \\
\hline Grossular & $\begin{array}{l}1003 \\
820\end{array}$ & & 876 & $\begin{array}{l}625 \\
502 \\
474\end{array}$ & 411 & 544 & & 325 & 372 & & 178 & $\begin{array}{l}243, \\
275\end{array}$ & \\
\hline Andradite & $\begin{array}{l}990 \\
839 \\
812\end{array}$ & 870 & & $\begin{array}{l}548 \\
446\end{array}$ & $\begin{array}{l}572 \\
488\end{array}$ & 513 & $\begin{array}{l}322, \\
308\end{array}$ & 347 & 365 & & 170 & $\begin{array}{l}232, \\
261\end{array}$ & 293 \\
\hline
\end{tabular}

\subsection{Infrared Spectroscopy Features}

Infrared (IR) spectra of garnet end-members consist of 17 vibrational modes $\left(\mathrm{F}_{1 \mathrm{u}}\right.$ modes) whose assignment has been proposed [26,27]. However, incomplete sets (less than 17 modes) have been reported in many experimental IR studies, suggesting that some of the modes are characterized by low intensity $[1,20,28]$. In theory, there are 10 bands in the mid-infrared region (MIR, $4000-400 \mathrm{~cm}^{-1}$ ) with concentrations in the $1100-400 \mathrm{~cm}^{-1}$ range. The observed IR bands and average frequencies in the MIR range for all the garnets species examined here are shown in Figure 4 and listed in Table 5.

Bands B, C, and D arising from the higher energy vibrations appear in the $1100-700 \mathrm{~cm}^{-1}$ region and are assigned as asymmetric $\mathrm{Si}-\mathrm{O}$ stretching modes $\left[\mathrm{v}_{3}\left(\mathrm{SiO}_{4}\right)\right][17,19]$. The shoulder which frequency is higher than band B, named band A [27], was not visible in all of the examined garnet samples. The origin of band $\mathrm{A}$ is not clear [17]. It was considered as an overtone by some researchers [20].

Bands $\mathrm{E}, \mathrm{F}$, and $\mathrm{G}$ appearing in the $700-500 \mathrm{~cm}^{-1}$ region are assigned to the symmetric $\mathrm{Si}-\mathrm{O}$ bending mode $\left[v_{4}\left(\mathrm{SiO}_{4}\right)\right]$, and the band I (about $470-450 \mathrm{~cm}^{-1}$ ) is assigned to the asymmetric $\mathrm{Si}-\mathrm{O}$ bending mode $\left[\mathrm{v} 2\left(\mathrm{SiO}_{4}\right)\right]$. In previous studies, the intensity of examined band $\mathrm{E}$ of the pyrope samples was too low to be observed, which is different from other garnets $[17,28]$. Bands $\mathrm{H}$ and $\mathrm{J}$ arising from the lower energy vibrations in the $500-400 \mathrm{~cm}^{-1}$ region are assigned to translations of the $B$ cations, $T(B)$, in the octahedral site $[17,27]$.

The same as Raman spectra, the patterns of IR spectra of aluminosilicate garnets are different from those of andradite samples. The patterns of IR spectra of aluminosilicate garnets are similar, especially in the higher wavenumber region. In IR spectra, bands in the fingerprint region (lower wavenumber region, $680-380 \mathrm{~cm}^{-1}$ ) are often used to discriminate minerals, as well as the species of garnet minerals [27]. However, the behavior of bands below $500 \mathrm{~cm}^{-1}$ does not allow elucidation of the structural features [19], these bands will not be discussed in terms of the correlations with garnet species. In grossular species, there is a little amount of wavenumber shift in IR bands for samples SLS-2 (hessonite), SLS-20 (tsavorite), and SLS-24 (Mali garnet) relative to samples SLS-14 (hessonite), SLS-15 (hessonite), SLS-19 (tsavorite), and SLS-25 (Mali garnet) as shown in Figure 4. The phenomenon may be caused by Transverse Optical-Longitudinal Optical (TO-LO) splitting [27,28]. 

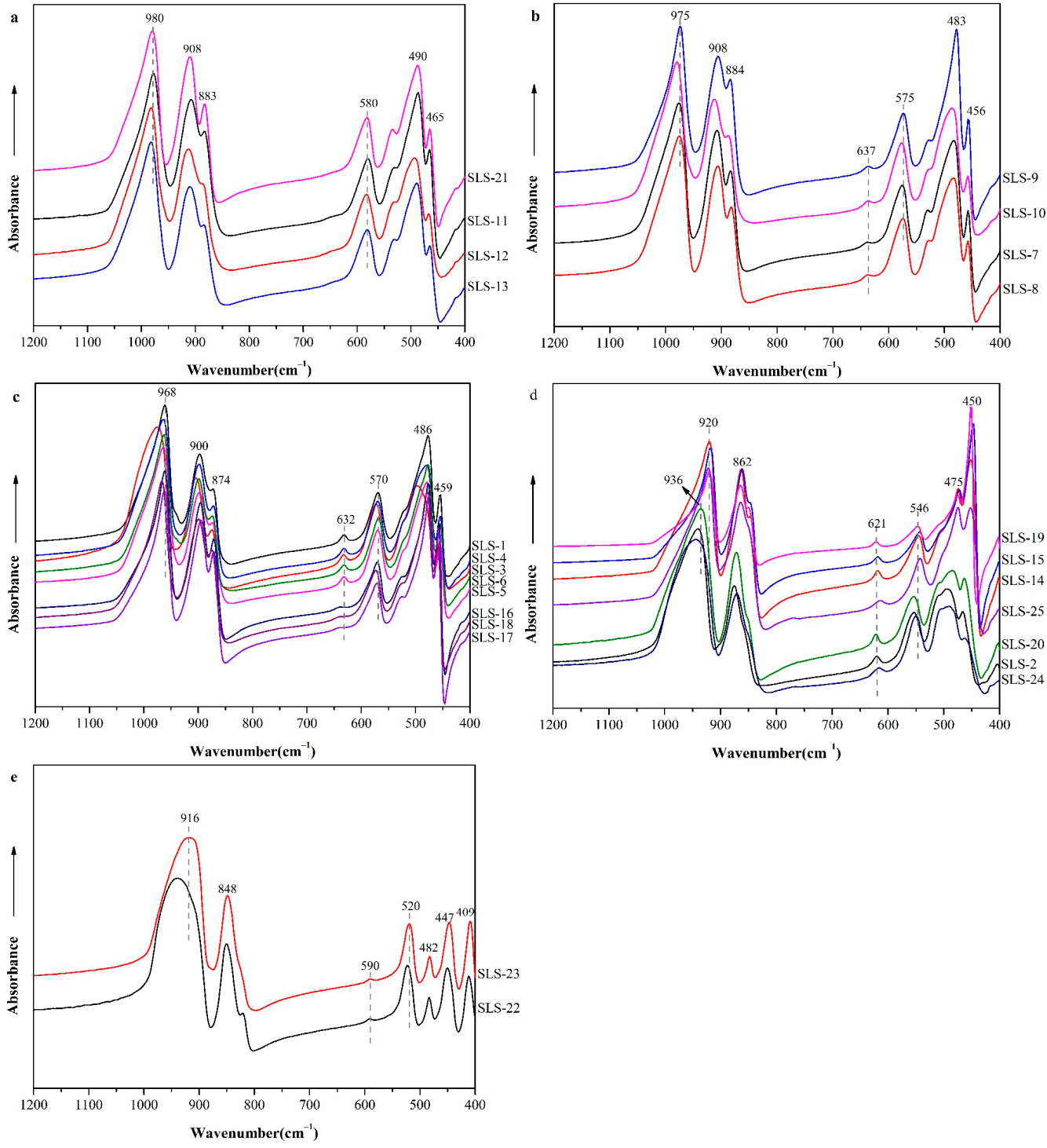

Figure 4. The Infrared spectra of gem garnet samples. (a) pyrope, (b) almandine, (c) spessartine, (d) grossular, and (e) andradite.

Table 5. Infrared absorbance band frequencies (in $\mathrm{cm}^{-1}$ ) for the studied garnets.

\begin{tabular}{|c|c|c|c|c|c|c|c|c|c|}
\hline \multirow[t]{2}{*}{ Species } & \multicolumn{3}{|c|}{$\begin{array}{c}\text { Asymmetric Stretching Vibration } \\
\text { of Si-O }\end{array}$} & \multicolumn{3}{|c|}{$\begin{array}{l}\text { Symmetric Bending } \\
\text { Vibration of Si-O }\end{array}$} & \multirow{2}{*}{$\begin{array}{c}\text { Asymmetric } \\
\text { Bending } \\
\text { Vibration } \\
\text { of Si-O }\end{array}$} & \multicolumn{2}{|c|}{ Lattice Vibration } \\
\hline & B & $\mathrm{C}$ & D & E & $\mathbf{F}$ & G & & $\mathbf{H}$ & $\mathbf{J}$ \\
\hline Pyrope & 980 & 908 & 883 & - & 580 & 529 & 465 & 490 & 417 \\
\hline Almandine & 975 & 908 & 884 & 637 & 575 & 529 & 456 & 483 & 415 \\
\hline Spessartine & $968 \pm 5$ & $900 \pm 5$ & 874 & 632 & 570 & 522 & 457 & $486 \pm 10$ & 417 \\
\hline Grossular & $940 \pm 3$ & $873 \pm 3$ & 847 & 621 & 554 & 508 & 463 & $490 \pm 5$ & 415 \\
\hline Andradite & $928 \pm 10$ & 848 & 820 & 590 & 520 & 482 & - & 447 & 409 \\
\hline
\end{tabular}

"-" peak or shoulder was not detected herein.

\section{Discussion}

Garnets present a relatively simple vibrational spectrum because of their high crystal symmetry [27]. Cation substitution on both the dodecahedral and octahedral sites of garnet is known to cause rotation of the tetrahedral site about its symmetry axis, variations of 
the $\mathrm{Si}-\mathrm{O}$ bond distances, changes in the unit cell volume dominates, and so on $[16,20,29]$. Therefore, the various garnet types caused by isotropic substitution permit the study of the correlations between the changing chemistry and vibrational spectra. It provides the opportunity to distinguish garnet varieties in Raman and IR spectra according to chemical changes without composition analysis. On the other hand, some gem properties of garnet, such as the values of SG and RI, also change because of chemical composition. Many studies had discussed the correlation between the IR or Raman spectra and cation substitutions in binary garnet samples [17-20]. However, the correlations among gemological properties, chemical composition, and infrared spectroscopy of the pyralspite and ugrandite series are only discussed by Adamo et al. (2007) [1].

In previous studies, it had been proposed that the type of cations, the cation radii, and mass of the A-site cations do not greatly affect the external mode frequencies of garnets but obviously affect internal stretching and bending vibrations of the $\mathrm{SiO}_{4}$-tetrahedra $[1,17,20]$. Therefore, in this IR and Raman spectra study, these vibrations of garnet are used to discuss the relationship between chemical changes and relative gem properties.

Figure 5 shows correlations among values of SG and RI, wavenumber shifts of stretching and bending vibrations, and five garnet species. The tested values of SG and RI of different garnet species exhibit mutually covered ranges and show no correlations between the type of cations in the A or B sites and gem properties. That is why they are not suitable for the precise identification of some garnet species. However, the frequencies of Raman $(\mathrm{Si}-\mathrm{O})_{\text {str }} \mathrm{F}_{2 \mathrm{~g}-},(\mathrm{Si}-\mathrm{O})_{\text {str }} \mathrm{A}_{1 \mathrm{~g}-}$ and $(\mathrm{Si}-\mathrm{O})$ bend $\mathrm{A}_{1 \mathrm{~g}-}$ modes and wavenumber of IR bands $\mathrm{B}$, $\mathrm{E}$, and $\mathrm{F}$ exhibit correlations with chemical change.

As well known, the order of the divalent cation radii in $\mathrm{A}$ site is $\mathrm{Mg}^{2+}<\mathrm{Fe}^{2+}<\mathrm{Mn}^{2+}<\mathrm{Ca}^{2+}$, and the order of the trivalent cation radii in $\mathrm{B}$ site is $\mathrm{Al}^{3+}<\mathrm{Fe}^{3+}$. The unit cell volumes of the end-member garnets increase in the following order: pyrope (1502.9 $\AA^{3}$, [30]), almandine (1530.8 $\AA^{3}$, [31]), spessartine (1565.7 $\AA^{3}$, [32]), grossular $\left(1640.9 \AA^{3}\right.$, [33]), andradite (1757.5 $\AA^{3}$, [34]). The relative atomic mass (Ar) of the constituent metals increases as follows: $\operatorname{Ar}(\mathrm{Mg})=24.31$, $\operatorname{Ar}(\mathrm{Al})=26.98, \operatorname{Ar}(\mathrm{Ca})=40.08, \operatorname{Ar}(\mathrm{Mn})=54.94$ and $\operatorname{Ar}(\mathrm{Fe})=55.85$.

For the five garnet species (Figure 5), the frequencies of Raman $(\mathrm{Si}-\mathrm{O})_{\text {str }} \mathrm{F}_{2 \mathrm{~g}-},(\mathrm{Si}-\mathrm{O})_{\mathrm{str}}$ $\mathrm{A}_{1 \mathrm{~g}-}$ and $(\mathrm{Si}-\mathrm{O})$ bend $\mathrm{A}_{1 \mathrm{~g}-}$ modes and wavenumber of IR bands $\mathrm{B}, \mathrm{E}$, and $\mathrm{F}$ decrease with increasing unit cell volumes of various garnets. Pyrope samples (rhodolite: SLS-11 13, rose-red garnet: SLS-21) have the highest frequencies and wavenumber of those modes and bands, while andradite samples (demantoid: SLS-22 and andradite: SLS-23) have the lowest frequencies and wavenumber. As in Raman spectra, the highest frequencies of (Si-O) $)_{\text {str }} \mathrm{F}_{2 \mathrm{~g}}$ mode is about $1046 \mathrm{~cm}^{-1}$ for pyrope, downshifted about $7 \mathrm{~cm}^{-1}$ for almandine, downshifted about $20 \mathrm{~cm}^{-1}$ for spessartine, and downshifted about $43 \mathrm{~cm}^{-1}$ for grossular, but just about $990 \mathrm{~cm}^{-1}$ for andradite (Figure 5 and Table 4). In IR spectra, the highest wavenumber of (Si-O) str band B is about $980 \mathrm{~cm}^{-1}$ for pyrope, downshifted about $5 \mathrm{~cm}^{-1}$ for almandine, downshifted about $12 \mathrm{~cm}^{-1}$ for spessartine, and downshifted about $40 \mathrm{~cm}^{-1}$ for grossular but just about $928 \mathrm{~cm}^{-1}$ for andradite (Figure 5 and Table 5). 


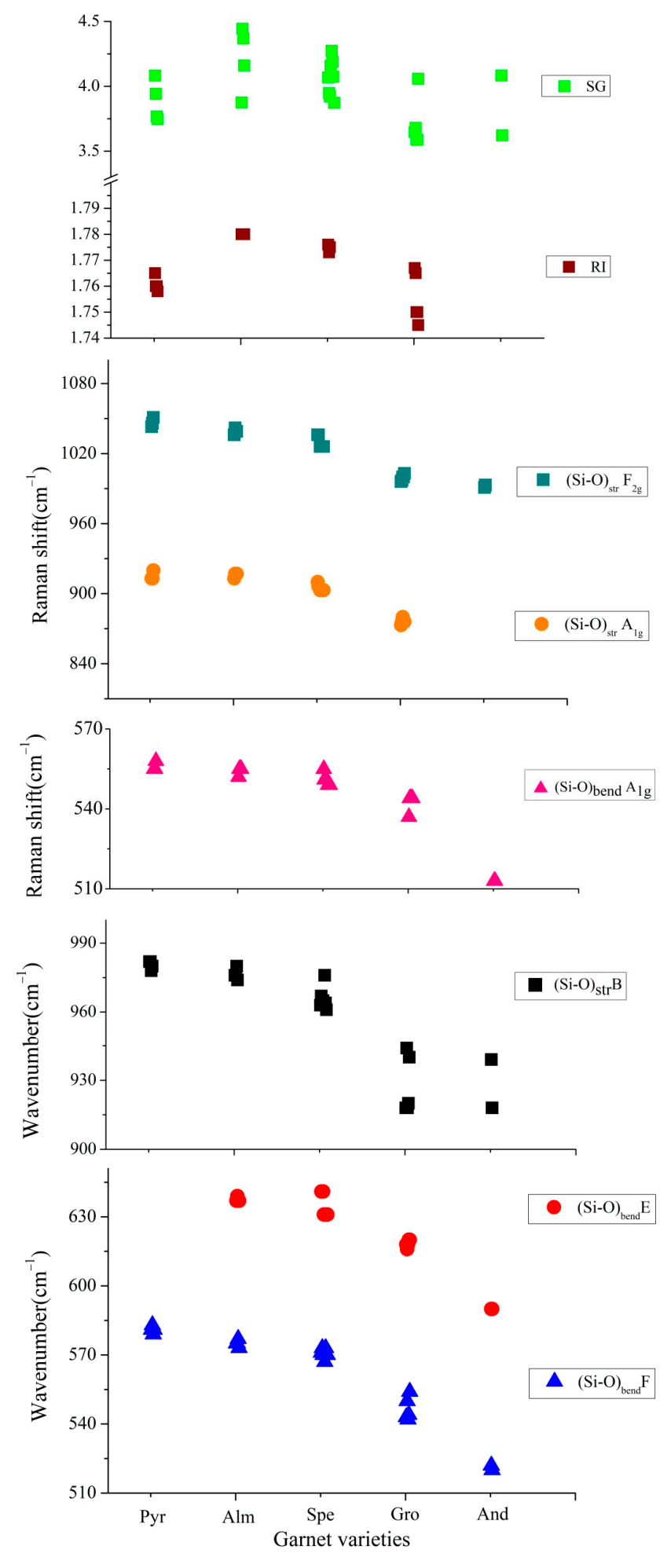

Figure 5. Correlations of SG values (yellowish-green circles), RI values (brown squares), Raman

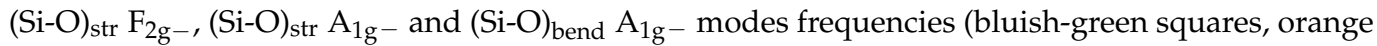
circles, and pink triangles) and IR B, E and F bands wavenumber (black squares, red circles, and blue triangles) with different garnet species. Pyr = pyrope, Alm = almandine, Spe = spessartine, Gro = grossular, and $=$ andradite .

For aluminosilicate garnets, the frequencies and wavenumber of $\mathrm{Si}-\mathrm{O}_{\text {str }}$ modes exhibit a close relationship with the dominant cations in A site. As shown in Figure 5 and Tables 4 and 5, the order of decreasing frequencies and wavenumber of those modes is the same as 
the increasing order of divalent cation radii and unit cell volumes but the peaks exhibit little relation with the relative atomic mass in a certain species. Such as in pyrope, correlation cannot be found between chemical compositions (e.g., pyr\%, or the mass of $\mathrm{Mg}$ cations, or the value of $\mathrm{Mg} /(\mathrm{Ca}+\mathrm{Fe}))$ and the frequencies of $(\mathrm{Si}-\mathrm{O})_{\mathrm{str}} \mathrm{A}_{1 \mathrm{~g}}$ mode or the wavenumber of (Si-O) str band B (Tables $2-5$ and Figures 3 and 4 ).

Andradite has the largest cations both in A site $\left(\mathrm{Ca}^{2+}\right)$ and in $\mathrm{B}$ site $\left(\mathrm{Fe}^{3+}\right)$ relative to those in aluminosilicate garnets and has relative the largest unit cell volume. It results in a sharp drop of frequencies and wavenumber of those modes and bands in Raman and IR spectra (Figure 5 and Tables 4 and 5). Therefore, it is easy to identify andradite according to the peak positions of $\mathrm{Si}-\mathrm{O}_{\text {str }}$ modes in Raman or in IR spectra. However, it needs to be noticed that the peak position at about $870 \mathrm{~cm}^{-1}$ in andradite belongs to $\mathrm{Si}-\mathrm{O}_{\mathrm{str}} \mathrm{E}_{\mathrm{g}}$ mode not $\mathrm{Si}-\mathrm{O}_{\text {str }} \mathrm{A}_{1 \mathrm{~g}}$ mode (Table 4).

When the combination of the Roman spectra and IR spectra can be used to identify the garnet species, the $1000-800 \mathrm{~cm}^{-1}$ region is assigned, as asymmetric $\mathrm{Si}-\mathrm{O}$ stretching modes can provide rapid and precise identification of the garnet species. The garnet species show obvious differences in the frequencies of the strongest peak ((Si-O $)_{s t r} \mathrm{~A}_{1 \mathrm{~g}}$ mode) and the following weak peak ((Si-O) $)_{s t r} F_{2 g}$ mode) in Roman spectra, and the bands $\mathrm{B}$ and $\mathrm{C}$ in IR spectra. For pyrope, the peaks are about $913 \mathrm{~cm}^{-1}$ and $860 \mathrm{~cm}^{-1}$ in Roman spectra, the bands are about $980 \mathrm{~cm}^{-1}$ and $908 \mathrm{~cm}^{-1}$ in IR spectra. For almandine, the peaks are about $916 \mathrm{~cm}^{-1}$ and $863 \mathrm{~cm}^{-1}$ in Roman spectra, the bands are about $975 \mathrm{~cm}^{-1}$ and $908 \mathrm{~cm}^{-1}$ in IR spectra. For spessartine, the peaks are about $906 \mathrm{~cm}^{-1}$ and $850 \mathrm{~cm}^{-1}$ in Roman spectra, the bands are about $968 \mathrm{~cm}^{-1}$ and $900 \mathrm{~cm}^{-1}$ in IR spectra. For grossular, the peaks are about $876 \mathrm{~cm}^{-1}$ and $820 \mathrm{~cm}^{-1}$ in Roman spectra, the bands are about $940 \mathrm{~cm}^{-1}$ and $873 \mathrm{~cm}^{-1}$ in IR spectra. For andradite, the strongest peak is (Si-O) str $E_{\mathrm{g}}$ mode (about $870 \mathrm{~cm}^{-1}$ ) followed by two weak (Si-O) str $F_{2 g}$ modes (about $839 \mathrm{~cm}^{-1}$ and $812 \mathrm{~cm}^{-1}$ ) in Roman spectra. Finally, in IR spectra, its bands B and C reduce to $916 \sim 928 \mathrm{~cm}^{-1}$ and $848 \mathrm{~cm}^{-1}$, respectively.

\section{Conclusions}

Chemical composition tests, such as electron microprobe analysis and LA-ICP-MS analysis, can provide precise specifications of types and series according to a full compositional characterization. However, it is less often used than IR and Raman spectroscopy in routine identification work in gem labs. Internal stretching vibrations of the $\mathrm{SiO}_{4}$-tetrahedra of garnet provide more precise identification of species than external modes because they have a correlation with the type of cations in the A or the B site and are closely related to the cation radii and the unit cell volumes. Therefore, a set of frequencies and wavenumber of $\mathrm{Si}-\mathrm{O}_{\mathrm{str}}$ modes obtained from Raman and IR spectroscopy can be used to quickly identify the garnet species without damaging the samples.

Supplementary Materials: The following supporting information can be downloaded at: https: / / www.mdpi.com/article/10.3390/cryst12010104/s1, Figure S1: The UV-Vis spectra of gem garnet sample.

Author Contributions: T.C. conceived the idea of the project, gave data interpretation, and drafted the manuscript. W.L. carried out experiments, wrote the original draft and gave data interpretation. J.Z. and X.X. carried out IR and Roman experiments. J.P. provided garnets samples. W.L. and T.C. contribute equally. All authors have read and agreed to the published version of the manuscript.

Funding: This research was funded by the National Natural Science Foundation of China, gran numbers 42072252 and the Fundamental Research Funds of Gemology Institute, China University of Geosciences, Wuhan, grant numbers CIGTXM-02-202002.

Institutional Review Board Statement: Not applicable.

Informed Consent Statement: Not applicable.

Data Availability Statement: All supporting data and computational details are available on written request. These data are stored by the main author of this article. 
Acknowledgments: We thank Zhaochu Hu's group for obtaining LA-ICP-MS data of garnet samples. We thank two anonymous reviewers for comments that improved our manuscript.

Conflicts of Interest: The authors declare no conflict of interest.

\section{References}

1. Adamo, I.; Pavese, A.; Prosperi, L.; Ajò, D. Gem-quality garnets: Correlations between gemological properties, chemical composition and infrared spectroscopy. J. Gemmol. 2007, 30, 307-313. [CrossRef]

2. Baxter, E.F.; Caddick, M.J.; Dragovic, B. Garnet: A rock-forming mineral petrochronometer. Rev. Mineral. Geochem. 2017, 83, 469-533. [CrossRef]

3. Grew, E.S.; Locock, A.J.; Mills, S.J.; Galuskina, I.O.; Galuskin, E.V.; Halenius, U. Nomenclature of the garnet supergroup. Am. Mineral. 2013, 98, 785-811. [CrossRef]

4. Antao, S.M. Crystal Chemistry of Six Grossular Garnet Samples from Different Well-Known Localities. Minerals 2021, 11, 767. [CrossRef]

5. Locock, A.J. An Excel spreadsheet to recast analyses of garnet into end-member components, and a synopsis of the crystal chemistry of natural silicate garnets. Comput. Geosci. 2008, 34, 1769-1780. [CrossRef]

6. Adamo, I.; Bocchio, R.; Diella, V.; Caucia, F.; Schmetzer, K. Demantoid from Balochistan, Pakistan: Gemmological and Mineralogical Characterization. J. Gemmol. 2015, 34, 428-433. [CrossRef]

7. Schwarzinger, C. Determination of demantoid garnet origin by chemical fngerprinting. Monatshefte Chem. Chem. Mon. 2019, 150, 907-912. [CrossRef]

8. Kos, S.; Dolenec, M.; Lux, J.; Dolence, S. Raman microspectroscopy of garnets from S-Fibulae from the Archaeological Site Lajh (Slovenia). Minerals 2020, 10, 325. [CrossRef]

9. Koralay, T.; Oren, U. Determination of spectroscopic features and gemstone potential of garnet crystals from the Camkoy region (Aydin-SW Turkey) using XRPD, XRF, Confocal Raman Spectroscopy, EPMA and gemological test methods. Mineral. Crystall. 2020, 89, 105-123.

10. Johnson, M.L.; Boehm, E.; Krupp, H.; Zang, J.W.; Kammerling, R.C. Gem-quality grossular-andradite: A new garnet from Mali. Gems Gemol. 1995, 31, 152-166. [CrossRef]

11. Phillips, W.R.; Ta1antsev, A.S. Russian Demantoid Czar of the Garnet Family. Gems Gemol. 1996, 32, 100-111. [CrossRef]

12. Krzemnicki, M.S.; Hanni, H.; Reusser, E. Colour change garnets from Madagascar: Comparison of colorimetric with chemical data. J. Gemmol. 2001, 27, 395-408. [CrossRef]

13. Hoskin, P.W.O.; Grapes, R.H.; Catchpole, H.; Klaudius, J. Horse-tail inclusions in demantoid garnet from Val Malenco, Italy. J. Gemmol. 2003, 28, 333-336. [CrossRef]

14. Schmetzer, K.; Bernhardt, H.J.; Kiefert, L. Star garnets and star garnet cat's-eyes from Ambatondrazaka, Madagascar. J. Gemmol. 2002, 28, 13-24. [CrossRef]

15. Teertstra, D.K. The refraction of light by garnet depends on both composition and structure. J. Gemmol. 2008, 31, 105-110. [CrossRef]

16. Kolesov, B.A.; Geiger, C.A. Raman spectra of silicate garnets. Phys Chem Miner 1998, 25, 142-151. [CrossRef]

17. Bosenick, A.; Geiger, C.A.; Schaller, T.; Sebald, A. A 29Si MAS NMR and IR Spectroscopic investigation of synthetic pyropegrossular garnet solid solutions. Am. Mineral. 1995, 80, 691-704. [CrossRef]

18. Du, W.; Han, B.F.; Clark, S.; Wang, Y.C.; Liu, X. Raman spectroscopic study of synthetic pyrope-grossular garnets: Structural implications. Phys. Chem. Miner. 2018, 45, 197-209. [CrossRef]

19. Makreski, P.; Runcevski, T.; Jovanovski, G. Minerals from Macedonia. XXVI. Characterization and spectra-structure correlations for grossular and uvarovite. Raman study supported by IR spectroscopy. J. Raman Spectrosc. 2011, 42, 72-77. [CrossRef]

20. Ballaran, T.B.; Woodland, A.B. Local structure of ferric iron-bearing garnets deduced by IR-spectroscopy. Chem. Geol. 2006, 225, 360-372. [CrossRef]

21. Droop, G.T.R. A general equation for estimating $\mathrm{Fe}^{3+}$ concentrations in ferromagnesian silicates and oxides from microprobe analyses, using stoichiometric criteria. Mineral. Mag. 1987, 51, 431-435. [CrossRef]

22. Stockton, C.; Manson, D. A proposed new classification for gem-quality garnets. Gems Gemol. 1985, 21, 205-218. [CrossRef]

23. Pezzotta, F.; Adamo, I.; Diella, V. Demantoid and topazolite from Antetezambato, Northern Madagascar: Review and new Data. Gems Gemol. 2011, 47, 2-14. [CrossRef]

24. Wu, F.; Zhang, X.C.; Zhu, Z.L. Quantitative relation between Raman shift and metal ion content in garnets. J. Light Scatt. 2015, 27, 350-354. (In Chinese)

25. Kanis, J.; Redmann, M. Four hessonite occurrences in Orissa, India. J. Gemmol. 1994, 24, 75-83.

26. Moore, R.K.; White, W.B.; Long, T.V. Vibrational spectra of the common silicates: I. The garnets. Am. Mineral. 1971, 56, 54-71.

27. Hofmeister, A.M.; Chopelas, A. Vibrational spectroscopy of end-member silicate garnets. Phys. Chem. Miner. 1991, 17, 503-526. [CrossRef]

28. Zicovich-Wilson, C.M.; Torres, F.J.; Pascale, F.; Valenzano, L.; Orlando, R.; Dovesi, R. Ab initio simulation of the IR spectra of pyrope, grossular, and andradite. J. Comput. Chem. 2008, 29, 2268-2278. [CrossRef] 
29. Woodland, A.B.; Ross II, C.R. A crystallographic and Mössbauer spectroscopy study of $\mathrm{Fe}_{3} \mathrm{Al}_{2} \mathrm{Si}_{3} \mathrm{O}_{12}-\mathrm{Fe}_{3}{ }^{2+} \mathrm{Fe}_{2}{ }^{3+} \mathrm{Si}_{3} \mathrm{O}_{12}$ (almandine-skiagite) and $\mathrm{Ca}_{3} \mathrm{Fe}_{2}{ }^{3+} \mathrm{Si}_{3} \mathrm{O}_{12}-\mathrm{Fe}_{3}{ }^{2+} \mathrm{Fe}_{2}{ }^{3+} \mathrm{Si}_{3} \mathrm{O}_{12}$ (andradite-skiagite) garnet solid solutions. Phys. Chem. Miner. 1994, 21, 117-132. [CrossRef]

30. Pavese, A.; Artioli, G.; Prencipe, M. X-ray single-crystal diffraction study of pyrope in the temperature range 30-973 K. Am. Mineral. 1995, 80, 457-464. [CrossRef]

31. Geiger, C.A.; Armbruster, T.; Lager, G.A.; Jiang, K.; Lottermoser, W.; Amthauer, G. A combined temperature dependent ${ }^{57}$ Fe Mössbauer and single crystal X-ray diffraction study of synthetic almandine: Evidence for the Gol'danskii-Karyagin effect. Phys. Chem. Miner. 1992, 19, 121-126. [CrossRef]

32. Novak, G.A.; Gibbs, G.V. The crystal chemistry of the silicate garnets. Am. Mineral. 1971, 56, 791-825.

33. Hazen, R.M.; Finger, L.W. Crystal structures and compressibilities of pyrope and grossular to 60 kbar. Am. Mineral. 1978, 63, 297-303.

34. Armbruster, T.; Geiger, C.A. Andradite crystal chemistry, dynamic X-site disorder and structural strain in silicate garnets. Eur. J. Mineral. 1993, 5, 59-71. [CrossRef] 